Peer review: This article has been subject to a double-blind peer review process

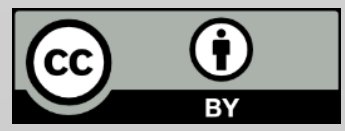

Copyright notice: This article is issued under the terms of the Creative Commons Attribution License, which permits use and redistribution of the work provided that the original author and source are credited.

You must give appropriate credit (author attribution), provide a link to the license, and indicate if changes were made. You may do so in any reasonable manner, but not in any way that suggests the licensor endorses you or your use. You may not apply legal terms or technological measures that legally restrict others from doing anything the license permits.

https://creativecommons .org/licenses/by/4.0/

\section{Electric Motor and Dry Clutch Control in Launch Manoeuvres of Mild-Hybrid Vehicles Based on AMT/DCT Transmissions}

\author{
Mario Pisaturo* and Adolfo Senatore
}

Department of Industrial Engineering, University of Salerno, Italy

*Correspondence: mpisaturo@unisa.it

\begin{abstract}
Mild-Hybrid Electric Vehicles (mild-HEVs) earned market share over the last years an as effective roadmap to limit air pollution in big cities. In addition to this role, hybrid propulsion can be used to avoid dry clutch overheating in mild-HEVs equipped with automated manual transmissions. Indeed, high thermal level could result in serious damaging of dry clutch linings with very fast decay of expected lifespan affecting vehicle reliability. This paper shows results of vehicle launch simulations to highlight how the propulsion due to electric motor can effectively reduce clutch thermal stress during the slipping phase.
\end{abstract}

Keywords: Hybrid electric vehicle; dry clutch; engagement control

\section{Introduction}

In the last decades, Hybrid Electric Vehicles (HEVs) and Electric Vehicles (EVs) are gaining significant share of the automobile market. Their success is mainly due to higher fuel efficiency than conventional vehicles which results also in a reduction of pollutant emissions.

By analysing the driveline structure, in parallel HEVs both the internal combustion engine (ICE) and electric motor (EM) can propel, separately or at the same time, the vehicle wheels. Series HEVs differ from parallel HEVs in the role of ICE as energy from combustion engine is converted into electric energy to charge the battery pack or to propel the wheels (Chan, 2007; Oh et al., 2007; Pisu \& Rizzoni, 2007).

The importance of moving toward sustainable mobility is strongly linked to the need to reduce air pollution and the greenhouse effect, especially in big cities. To this aim, many studies have been proposed to improve the efficiency of hybrid vehicles. 
In (Wang et al., 2015), dynamic programming global optimization algorithm is applied in HEV to search optimal solution in an assigned speed cycle that minimize the fuel consumption keeping the balance of battery state of charge (SOC). Moreover, in (Tribioli et al., 2014) a real-time energy management strategy for plugin HEVs based on optimal control theory is proposed.

In (Ye et al., 2013), a comprehensive control scheme, including energy management and coordinated control of parallel hybrid electric bus with automated manual transmission (AMT) based on dry clutch is presented. Instead, in (Vagg et al., 2016) a stochastic dynamic programming (SDP) is applied to optimal control of HEV.

The electric motor (EM) can even play a crucial role to reduce the thermal stress in dry clutch automated manual transmission (AMT) and dual clutch transmission (DCT) during the slipping phase by avoiding critical values (Pisaturo \& Senatore, 2017; Pisaturo, Senatore \& D’Agostino, 2017). Indeed, the most important problem with dry clutch systems like AMT and DCT is the overheating due to repeated shifting (Pica et al., 2016; Pisaturo \& Senatore, 2016) that results in poor quality engagements or even permanent damage of clutch frictional materials or facings. Thus, the accuracy of clutch temperature estimation deeply affects the control unit decisions. This paper is an extended version of (Pisaturo \& Senatore, 2017) but conversely from it, in this paper a more complex thermal model has been implemented in the transmission control unit (TCU) to activate the EM and reduce thermal stress on clutch components. Indeed, the advantage to have a complex thermal model is to better predict the transmitted clutch torque and consequently the improvement of the passenger comfort together to the extension of clutch life. Furthermore, the actuators' delay have been considered in the Model Predictive Control (MPC) design in the choice of both control and prediction to improve control performances. Particularly, critical launch manoeuvres with high initial clutch material temperature are taken into account. The results highlight how the activation of EM reduces the thermal power generated during the engagement manoeuvre by inhibiting clutch damage.

\section{Parallel HEV Driveline}

\section{Driveline model}

This section describes the driveline model used for simulating the parallel HEV longitudinal dynamics, where $T$ indicates the torques, J the inertias and $b$ the damping coefficients. Moreover, the subscripts e, f, c, g, d, m, w indicate engine, flywheel, clutch disc, gearbox, differential, electric motor and wheels, respectively. The scheme of the analysed driveline under the 
hypothesis of rigidity of all the shafts is reported in Figure 1 and the related equations are:

$$
\begin{gathered}
J_{e f} i_{\ldots}{ }_{2} \omega_{e}-T_{f c} \\
J_{v i} \ldots_{\ldots, j}+s r_{2} r_{d} T_{m}-\left(b_{g}\left(r_{1} r_{d}\right)^{2}+s b_{m}\left(r_{2} r_{d}\right)^{2}\right) \omega_{w}-T_{w}
\end{gathered}
$$

where $r_{1}, r_{2}$ and $r_{d}$, are the gear ratios on the engine side, electric motor side and differential respectively. Moreover, $s$ is a parameter used to switch between the HEV mode $(s=1)$ and pure ICE mode $(s=0)$. In addition, the following positions hold:

$$
\begin{aligned}
& J_{e f}=J_{e}+J_{f} \\
& J_{v}=J_{w}+J_{d}+\left(J_{g 2}\right) r_{d}^{2}+\left(J_{g 1}+J_{c}\right)\left(r_{1} r_{d}\right)^{2}+\left(J_{g 3}+s J_{m}\right)\left(r_{2} r_{d}\right)^{2} \\
& T_{w}=m g R_{w}(\sin (\phi)+f \cos (\phi))+\frac{1}{2} \rho A C_{x} v^{2} R_{w}+T_{\text {brake }}
\end{aligned}
$$

where, $m$ is the vehicle mass, $R_{w}$ is the wheel radius, $\phi$ is the road slope, $f$ is the rolling resistance coefficient, aria $\rho$ is the air density, $A$ is the vehicle frontal area, $C_{x}$ is the air drag coefficient, $v$ the vehicle speed and $T_{\text {brake }}$ is the braking torque.

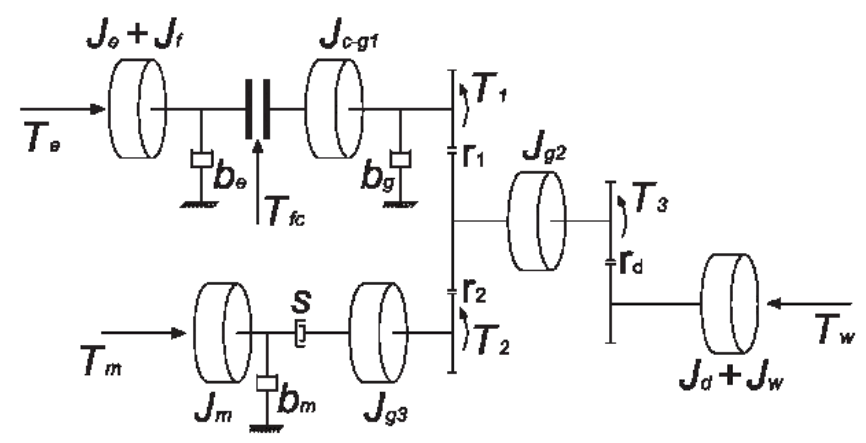

Figure 1 Driveline model.

The equation related to the "locked-up" model can be easily achieved from eqs. (1) and (2) by taking into account driveline dynamics reduced to engine (or wheel) shaft.

\section{Internal combustion engine model}

The engine behaviour has been implemented through a static torque map as function of engine speed ("plant" output) and accelerator pedal as well. Particularly, the first MPC output given by the reference engine torque is used to achieve the accelerator pedal signal by way of engine map inversion. Moreover, the delay introduced by the so-called engine torque build-up is modelled in all the performed simulations. Such an effect is related to the transient response turbocharger in modern automotive and truck diesel engines to attain steady-state conditions since optimization as 
design key-factor is based on low specific consumption in stationary states (Rakopoulos \& Giakoumis, 2009).

\section{Models for Dry Clutch and Electric Motor}

\section{Dry clutch model}

In (Vasca et al., 2011), a model of frictional torque in dry clutch based AMT gearbox is proposed. The paper underlines the role of the cushion spring load-deflection characteristic as well as its influence on clutch torque transmission. The model is depicted by neglecting the thermal effects. Instead, in (D'Agostino, Senatore \& Pisaturo, 2013) the Authors proposed a more complex transmissibility model which covers thermal effects both on cushion spring and clutch frictional response:

$$
T_{f c}\left(x_{t o}, \theta_{f s}, \theta_{c m}, v_{s}, p\right)=n \mu\left(v_{s}, p, \theta_{c m}\right) R_{m} F_{f c}\left(\delta_{f}\left(x_{p p}\left(x_{t o}, \theta_{f s}\right), \theta_{f s}\right)\right)
$$

where $\mathrm{T}_{\mathrm{fc}}$ is the transmitted clutch torque, $x_{\mathrm{to}}$ is the throwout bearing position, $\theta_{\mathrm{fs}}$ and $\theta_{\mathrm{cm}}$ are the cushion spring and clutch material temperature respectively. $v_{s}=R_{m} \omega_{s l}$ is the linear sliding speed, $R_{m}$ is the mean radius, $\omega_{s l}=\left|\omega_{e}-\omega_{c}\right|$ is the difference between the engine and clutch angular speeds, $\mathrm{p}$ is the contact pressure, $\mathrm{n}$ is the number of friction surfaces, $\mu$ is the friction coefficient, $F_{f c}$ is the clamping force, $\delta_{f}$ is the cushion spring deflection and $\mathrm{x}_{\mathrm{pp}}$ is the pressure plate position.

The clutch torque model is coupled to the temperature rise equation through the cushion spring load-deflection characteristic and frictional material response as well. The clutch actuator dynamics is given by discrete-time model with unitary gain first-order transfer and $0.1 \mathrm{~s}$ time constant. Thus, the reference clutch torque, i.e. the second output of the controller is inverted by a simplified clutch model to provide as output the reference throwout bearing position ("position controlled clutch"). The latter signal as input of the clutch actuator allows the calculation of the actual clutch torque in a detailed clutch torque map ( $n-D$ look-up table).

By considering the clutch transmissibility model described in equation (4), it is clear that an estimation of both clutch facing and cushion spring temperature is necessary. For this reason, a lumped thermal model has been implemented to take the temperature rise into account during the slipping phase. The model is inspired to the thermal model proposed in (Pica et al., 2016) whereas in the present paper the cushion spring response to the temperature change is modelled in addition to frictional material response. The main hypothesis is that the whole mechanical work is converted into heat by friction phenomena and that it is equally distributed onto the frictional surfaces. Moreover, when clutch is open, i.e. $\beta=0$, only convective losses take place whereas when clutch is in the slipping phase, i.e. $\beta=1$, only conductive phenomena occur and 
convective losses are negligible on cushion spring and clutch material. On the contrary, the body convective losses cannot be neglected when the clutch is closed. For this reason, the term $(2-\beta)$ accounts for about doubled clutch surface active to convective heat exchange when clutch is open. Thus, the thermal dynamics of clutch material $\theta_{\mathrm{cm}}$, body $\theta_{\mathrm{b}}$ and cushion spring $\theta_{\mathrm{fs}}$ is provided by first order differential equations:

$$
\begin{aligned}
& \left.i_{b} \quad \cdots,{ }_{c m}-\theta_{b}\right)-\alpha_{2}(2-\beta)\left(\theta_{b}-\theta_{h}\right)+\alpha_{3} \frac{T_{f c} \omega_{s l}}{2} \\
& i_{c m} \quad \ldots, r s\left(\theta_{c m}-\theta_{b}\right)-\alpha_{5}(1-\beta)\left(\theta_{c m}-\theta_{h}\right)-\alpha_{6} \beta\left(\theta_{c m}-\theta_{f s}\right)+\alpha_{7} \frac{T_{f c} \omega_{s l}}{2} \\
& i_{f s} \quad-\cdots \gamma\left(\theta_{c m}-\theta_{f s}\right)-\alpha_{9}(1-\beta)\left(\theta_{f s}-\theta_{h}\right)
\end{aligned}
$$

where $\theta_{\mathrm{h}}$ is the ambient temperature assumed constant and $\alpha_{\mathrm{i}}$ are the thermal parameters which need to be identified.

Due to the difficulties and costs to measure both the interface temperature and cushion spring temperature in a real system, results from Finite Element Analysis (FEA) (Pisaturo \& Senatore, 2016) have been used to estimate the thermal parameters ai. Particularly, the same thermal power used as energy source in FEA model has been fed as input of thermal model described above.

Furthermore, both clutch material and cushion spring reference temperatures used for parameter estimation have been calculated at mean radius. For the sake of brevity, the readers can find details on thermal parameters, on thermal power input and how the latter has been calculated for the three analysed launch manoeuvres in (Pisaturo \& Senatore, 2016).

Finally, In Figure 4 the mean and standard deviation of each parameter has been plotted. It is worth noting that, the main influencing phenomenon is the thermal conduction between clutch material and pressure plate (flywheel). Indeed, the two parameters 1 and 4 have the greatest values. Particularly, the parameter 4 features both the highest value and the highest uncertainty. 


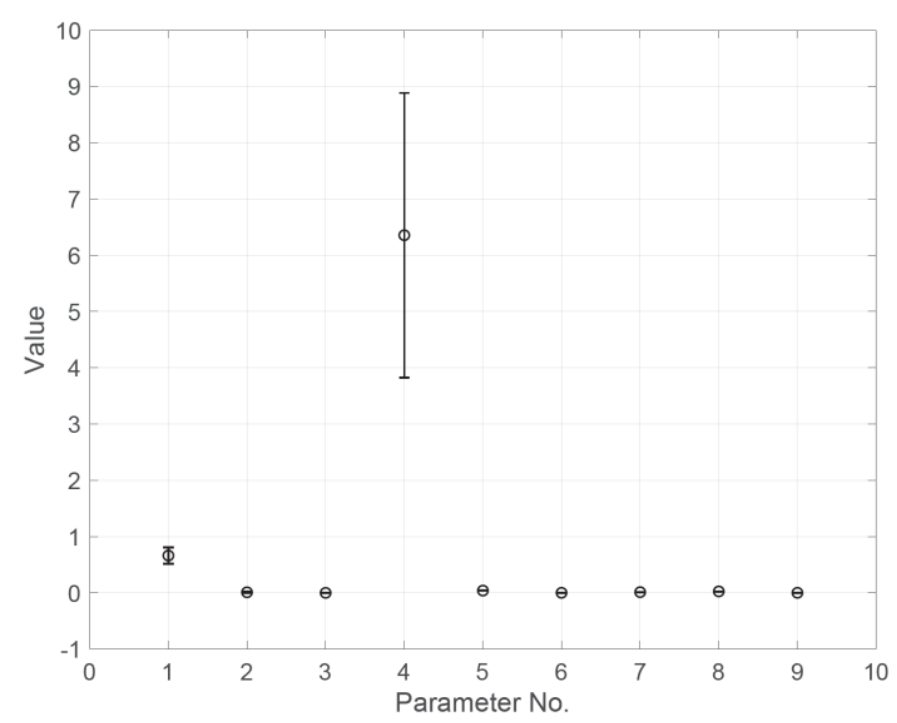

Figure 2 Estimated parameters: mean and standard deviation.

\section{Electric motor model}

As claimed before, this study aims at investigating the beneficial role of EM to limit temperature rise in dry clutch during HEV launch manoeuvre. Indeed, the EM activation enables torque delivery to traction wheels to accelerate the vehicle. In a simplified analysis, the EM dynamics does not involve any efficiency map to keep clear the benefit from EM activation and prevent complexity given by the coupling between clutch and EM thermal dynamics. In particular, a simple unitary gain, first-order transfer function with a time constant equal to $0.05 \mathrm{~s}$ has been used. In this way, the helpful role of the EM can be easily and directly compared to pure ICE mode.

According to the simulated driveline architecture, EM acts as an actuator which delivers the requested torque (third MPC output) to the driveline. In addition, the ICE power to drive the vehicle and charge the battery (motor becomes generator); the stationary charging mode and the regenerative braking mode have not been simulated in the framework of this paper. As future work, more inclusive HEV models will be analysed to encompass thermal management of driving motor and battery, as tradeoff with thermal relief of clutch in EM control strategy.

\section{Control Unit}

In Figure 3, the control scheme is reported. The MPC generates the reference signals for engine, clutch and electric motor subsystems. Particularly, the reference engine torque (first MPC output), together with the measured engine angular speed; feed an inverted engine map in order to obtain a reference accelerator pedal signal. Instead, the reference clutch torque (second MPC output) is inverted by means of clutch model 
to get the reference throwout bearing position. This signal, coupled to clutch actuator dynamics, has been used as an input to the detailed clutch torque transmissibility model, equation (4), to obtain the actual clutch torque signal ("plant" input). Finally, the reference electric motor torque (third MPC output) feed a unitary gain first-order transfer function with a time constant equal to $0.05 \mathrm{~s}$ which represents the electric motor dynamics.

To estimate the temperature rise during the engagement process, a thermal model based on equation (5) has been implemented in the control unit. The thermal model is fed with the reference clutch torque (second MPC output) and the measured engine and clutch angular speeds. Indeed, in automotive applications both the engine and wheel angular speeds, $\omega_{e}$ and $\omega_{w}$, as well as the road load $T_{w}$ are measured, (Sharifzadeh et al., 2016).

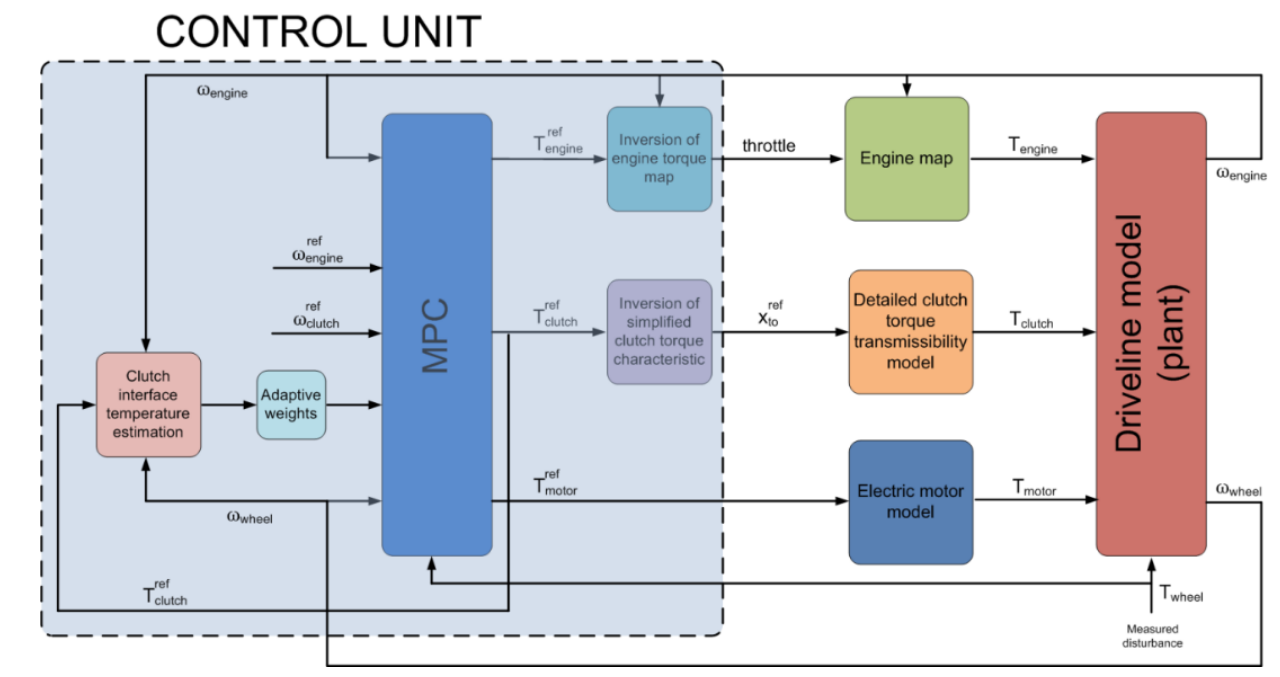

Figure 3 Control scheme.

\section{Model Predictive Control Design}

In this section, the model predictive control approach based on the work proposed in (Pisaturo, Cirrincione \& Senatore, 2015) is depicted. The controller has been designed with the discrete time version of the driveline model by assuming a sampling time of $0.01 \mathrm{~s}$ and the zero-order hold method. This value is compatible with automotive current technology requirements (Huang, Song \& Li, 2011).

Many studies pointed out that the higher thermal stress on clutch components is generated during the start-up manoeuvre whereas it is negligible during gear-shifts. For this reason, the driveline state-space model has been obtained by considering only the first gear. Moreover, all non-linarites such as the clutch and engine dynamics have been considered out of the MPC as look-up tables. Moreover, actuator delays 
have been considered in the MPC design to improve controller performances.

The state-space representation used to design the MPC is reported below:

$$
\left\{\begin{array}{l}
\mathbf{x}_{k+1}=\mathbf{A} \mathbf{x}_{k}+\mathbf{B}_{M V} \mathbf{u}_{k-\tau(k)}+\mathbf{B}_{M D} \mathbf{v}_{k} \\
\mathbf{y}_{k}=\mathbf{C} \mathbf{x}_{k}
\end{array}\right.
$$

where $\mathbf{x}=\left[\omega_{e} \omega_{w}\right]^{\top}$ is the state vector, $\mathbf{y}=\left[\omega_{e} \omega_{c}\right]^{\top}$ is the output vectors and $\mathbf{u}=\left[T_{e} T_{f c} T_{m}\right]^{\top}$ is the manipulated variables vector, $\mathbf{v}=\left[T_{w}\right]$ is the measured disturbance vector and $\omega_{e}$ and $\omega_{w}$ are the measured outputs of the controller.

The state matrix is $\mathbf{A}=\left[\begin{array}{cc}-\frac{b_{e}}{J_{e f}} & 0 \\ 0 & -\frac{b_{g}\left(r_{1} r_{d}\right)^{2}}{J_{v}}\end{array}\right]$, the input matrix is $\mathbf{B}=\left[\begin{array}{ll}\mathbf{B}_{\mathbf{M V}} & \mathbf{B}_{\mathbf{M D}}\end{array}\right]=\left[\begin{array}{cccc}\frac{1}{J_{e f}} & -\frac{1}{J_{e f}} & 0 & 0 \\ 0 & \frac{r_{1} r_{d}}{J_{v}} & s \frac{r_{2} r_{d}}{J_{v}} & -\frac{1}{J_{v}}\end{array}\right]$ and the output matrix is $\mathbf{C}=\left[\begin{array}{cc}1 & 0 \\ 0 & r_{1} r_{d}\end{array}\right]$.

\section{Plant constraints}

Some constraints both on the "plant" input and output have been selected to design the MPC and avoid the engine stall condition and provide comfortable lock-up at the same time. On the "plant" input saturation constraints have been imposed both on the torques:

$$
\begin{gathered}
-82 \leq T_{e} \leq 334 \quad \mathrm{Nm} \\
0 \leq T_{f_{c}} \leq 400 \quad \mathrm{Nm} \\
0 \leq T_{m} \leq 140 \quad \mathrm{Nm}
\end{gathered}
$$

where $-82 \mathrm{Nm}$ and $334 \mathrm{Nm}$ are the minimum and maximum engine torque values obtained from the engine static map. Moreover, $0 \mathrm{Nm}$ and $400 \mathrm{Nm}$ are the minimum and maximum torque values that the clutch can transmit. The maximum value has been assumed $20 \%$ greater than maximum engine torque. Finally, $0 \mathrm{Nm}$ and $140 \mathrm{Nm}$ are the minimum and maximum torque values that the electric motor can generate. As introduced before, in this study only the pure electric mode has been considered and the regenerative phase has not simulated.

Besides, to avoid the engine stall condition and guarantee comfortable lock-up, some constraints have been adopted also on "plant" outputs, i.e. engine and clutch angular speeds. 


$$
\begin{gathered}
80 \leq \omega_{e} \leq 500 \mathrm{rad} / \mathrm{s} \\
\omega_{c} \geq 0 \mathrm{rad} / \mathrm{s}
\end{gathered}
$$

where $80 \mathrm{rad} / \mathrm{s}$ represents the so-called no-kill condition (Glielmo et al., 2006), $500 \mathrm{rad} / \mathrm{s}$ is the maximum value of the engine speed before attaining critical conditions. Also these values have been obtained from the engine static map. Finally, $0 \mathrm{rad} / \mathrm{s}$ is the minimum clutch angular speed imposed to avoid reverse motion. It is worth noting that it is not necessary to impose a maximum clutch angular speed, because it is equal to the engine angular speed during the engaged phase and it can only decrease for passive resistance during the idle phase.

\section{Optimization problem and tuning}

The goal of the optimization problem is to minimize a cost function while satisfying constraints at each time step. As previously explained, the actuator delays have been considered in the MPC design. This affects the choice of both control and prediction horizon as follows:

$$
p-m>\frac{\max \left(\tau_{c}, \tau_{m}\right)}{T_{s}}
$$

where $p$ is the prediction horizon, $m$ is the control horizon, $\tau_{c}$ and $\tau_{m}$ are the clutch and electric motor actuator delays respectively and $T_{s}$ is the sampling time. As mentioned above, the clutch actuator delay is $0.1 \mathrm{~s}$ and the sampling time is $0.01 \mathrm{~s}$, thus the implemented values are $\mathrm{p}=25, \mathrm{~m}=$ 1. The implemented cost function to be minimizes is:

$$
F=\left[\mathbf{y}_{j}-\mathbf{r}_{j}\right]^{T} \mathbf{W}_{y, j}^{2}\left[\mathbf{y}_{j}-\mathbf{r}_{j}\right]+\left[\mathbf{u}_{i}-\mathbf{u}_{t a \arg e t, i}\right]^{T} \mathbf{W}_{u, i}^{2}\left[\mathbf{u}_{i}-\mathbf{u}_{t \operatorname{arget}, i}\right]+\Delta \mathbf{u}_{i}^{T} \mathbf{W}_{\Delta u, i}^{2} \Delta \mathbf{u}_{i}
$$

where $\mathbf{u}_{\mathrm{i}}$ is the input vector, $\mathbf{u}_{\text {target, } i}=\left[0,0, T_{\text {m,max }}\right]$ is the input target vector, $\Delta \mathbf{u}_{i}$ is the input increment vector, $\mathbf{y}_{i}$ is the output vector, $\mathbf{r}_{i}$ is the set point trajectory vector. Finally, the subscript $i$ and $j$ take into account the $i$-th inputs and j-th outputs of the "plant" respectively.

According to working conditions, adjustable weights for "plant" inputs and outputs have been implemented to trigger on the electric motor when the estimated clutch temperature reaches the threshold value of $200{ }^{\circ} \mathrm{C}$. Particularly, if the clutch temperature is lower than $200{ }^{\circ} \mathrm{C}$ the input weights are $\mathbf{W}_{\mathrm{u}}=\operatorname{diag}(0,0,0)$. On the contrary, when the clutch temperature overcomes $200^{\circ} \mathrm{C}$ the input weights are $\mathbf{W}_{\mathrm{u}}=\operatorname{diag}(0,0,5)$. In this way, to minimize the second term of the cost function, the difference between the input vector and the input target vector should be zero, i.e. $\mathrm{T}_{\mathrm{m}}{ }^{\text {ref }}=\mathrm{T}_{\mathrm{m} \text {,max. }}$. It is worth noting that the weights of the first two inputs are zero. This means that their difference from the target values does not 
affect the cost function. Finally, constant weights have been assumed for input increment $\mathbf{W}_{\Delta \mathrm{u}}=\operatorname{diag}(0.15,0.10,0.20)$ and output $\mathbf{W}_{\mathrm{y}}=\operatorname{diag}(1,1)$.

\section{Simulation Results}

In this section, two scenarios of vehicle launch manoeuvre simulating heavy working conditions are presented. Particularly, in the first scenario, an initial temperature of $190{ }^{\circ} \mathrm{C}$ as a result of previous engagements is assumed whereas an initial temperature of $210^{\circ} \mathrm{C}$ in the second scenario. Moreover, for both scenarios a road slope of 10 degree has been considered. In such circumstances, the amount of heat produced during the start-up manoeuvre could result in clutch material drawback. Moreover, repeated uphill launch makeovers in crowded traffic conditions, namely repeated clutch engagement in short time, are tangible danger to attain high interface temperatures and consequently clutch facings damage (Feng, Yimin and Juncheng, 2010). At this end, custom Matlab/Simulink model which takes into account a detailed dry clutch torque transmissibility model (i.e. by considering cushion spring thermal expansion and temperature-pressure-sliding speed dependent friction coefficient) has been implemented to simulate the longitudinal vehicle dynamics.

As previously explained, the control strategy penalizes the electric motor activation if the estimated clutch material temperature is less than $200{ }^{\circ} \mathrm{C}$. In Figures 4, 5 and 6, results of the analysed launch manoeuvre are reported in pure ICE mode and HEV mode. With reference to the latter case, in Figure 4 is highlighted as after about $0.7 \mathrm{~s}$ the clutch angular speed slope is higher than the pure ICE mode. This is due to the electric motor activation which delivers more torque to the traction wheels, see Figure 5. This results in a decrease of both engine and clutch torque and consequently in a reduction of the thermal power generated during the slipping phase. Moreover, as shown in Figure 6, the activation temperature threshold is reached and there is the transition from pure ICE to hybrid propulsion, i.e. $T_{m}>0$ (red line). Finally, in Figure 6 is shown as in the HEV mode clutch peak temperature is about $7{ }^{\circ} \mathrm{C}$ lower than the ICE mode. 


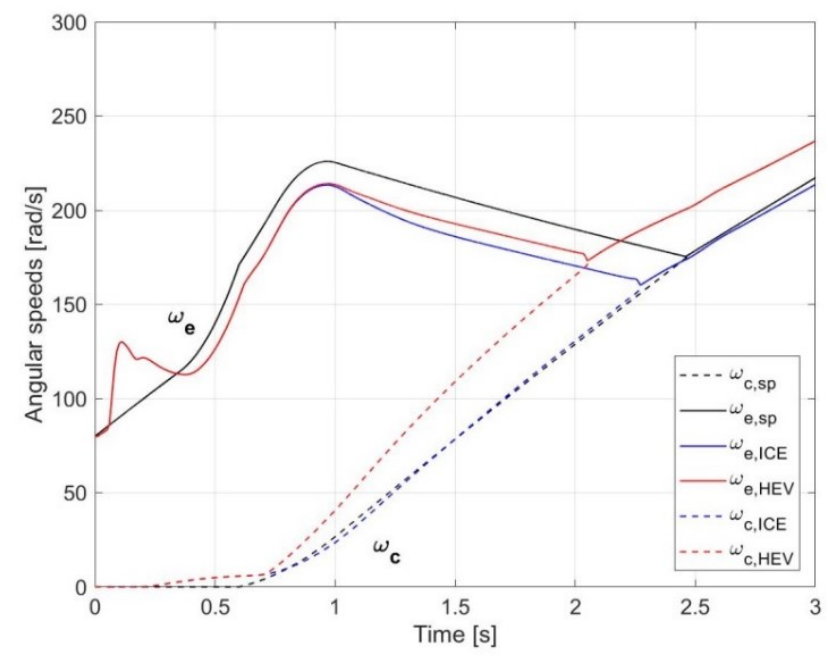

Figure 4 First scenario: engine and clutch angular speeds, set point trajectories (black lines), HEV mode (red lines), ICE mode (blue lines).

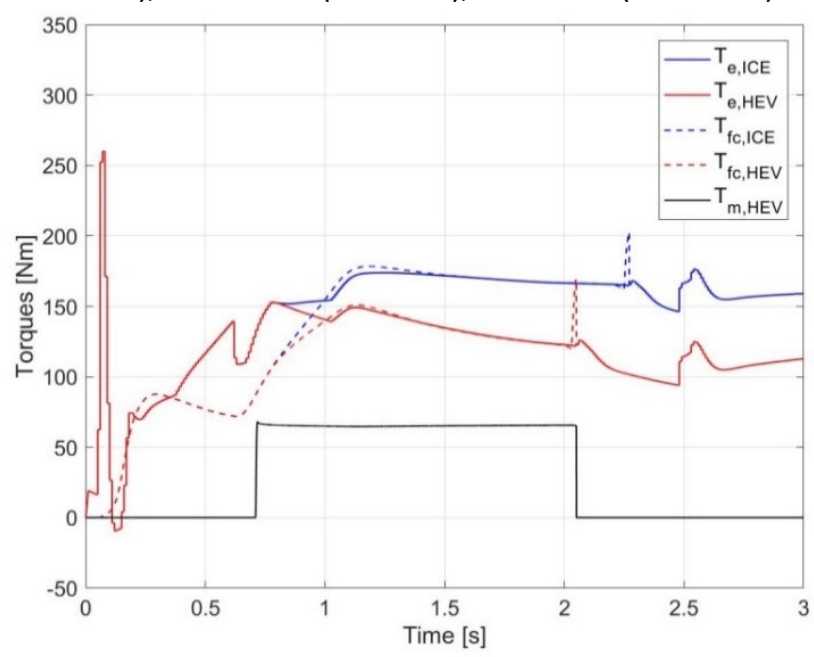

Figure 5 First scenario: engine, clutch and electric motor torques reduced to the engine shaft, HEV mode (red lines), ICE mode (blue lines) and electric motor torque (black line).

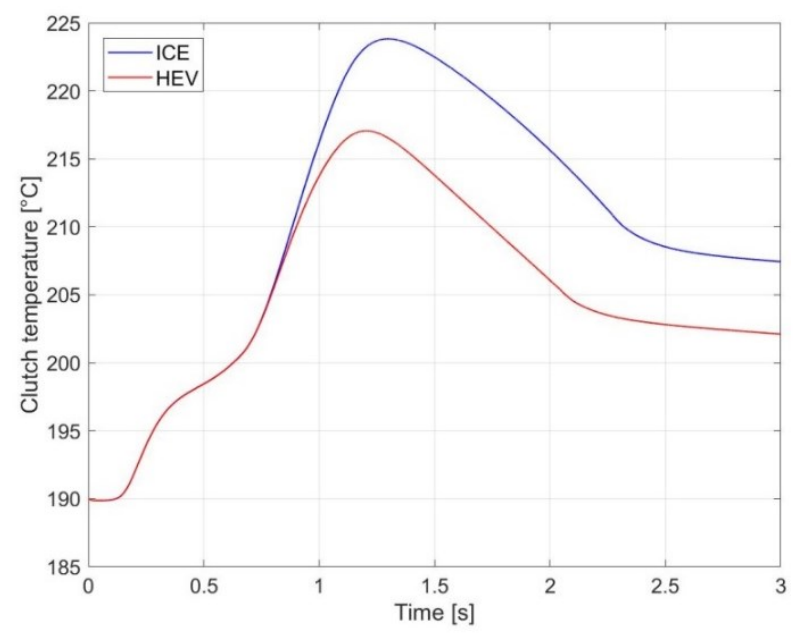

Figure 6 First scenario: clutch material temperature, HEV mode (red line), ICE mode (blue line). 
In the second scenario, an initial clutch temperature equal to $210{ }^{\circ} \mathrm{C}$ is selected; so, the activation temperature has already been overcome after previous clutch operations. With the same arrangement of the figures as for previous case, the first obvious result is the switching-on of the electric motor ( $T_{m}>0$ in Figure 8 ) since the first instants, as the MPC control rapidly triggers the electric propulsion to comply with the discussed temperature onset. The clutch torque spikes at the end of the engagement phase, highlighted in Figure 5 and 8, occur because the difference between the engine and clutch angular speeds are lower than the threshold at which the clutch is considered locked-up. Consequently, the TCU drives rapidly the clutch actuator to its rest position. This could results into longitudinal vibrations but by adapting the threshold it is possible to reduce them. In our case, the aim is to reduce the clutch damage due to thermal stress thus slightly higher vibrations are accepted.

In Figure 9, it is shown the clutch material temperature rising around $33^{\circ} \mathrm{C}$ in ICE mode, whereas the temperature rise in the same manoeuvre in HEV mode is only $18{ }^{\circ} \mathrm{C}$ : i.e. about $45 \%$ less than the clutch temperature increase in ICE mode.

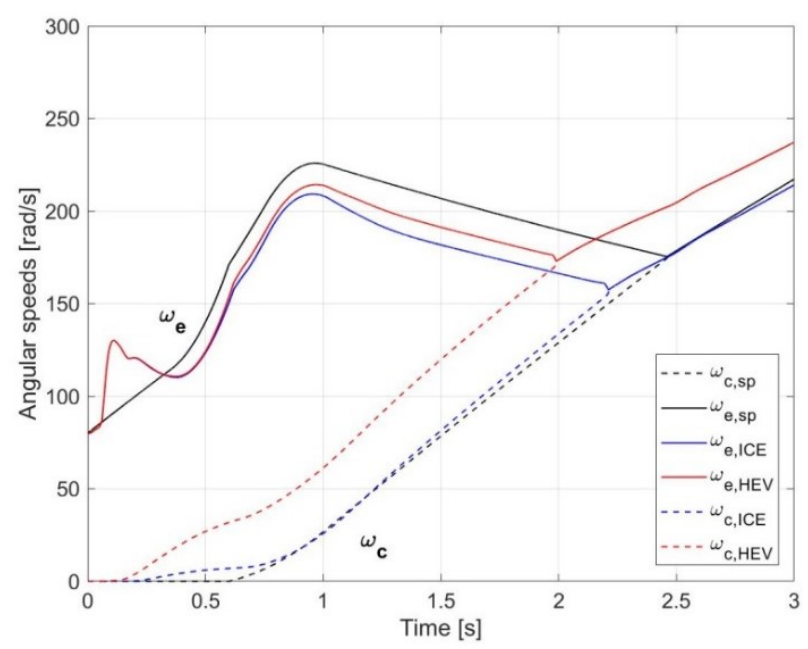

Figure 7 Second scenario: engine and clutch angular speeds, set point trajectories (black lines), HEV mode (red lines), ICE mode (blue lines).

\section{Conclusions}

This paper explores the benefits achievable in HEVs equipped with automated manual transmissions in preventing dry-clutch system from overheating problems. At this end, an investigation on the role of EM to minimize temperature rise in dry clutch AMT/DCT during the slipping phase is proposed. Simulations of start-up manoeuvre under hypothesis of initial temperature of $190^{\circ} \mathrm{C}$ or $210^{\circ} \mathrm{C}$ and road slope of 10 degree with and without electric propulsion aid have been carried out. The results 


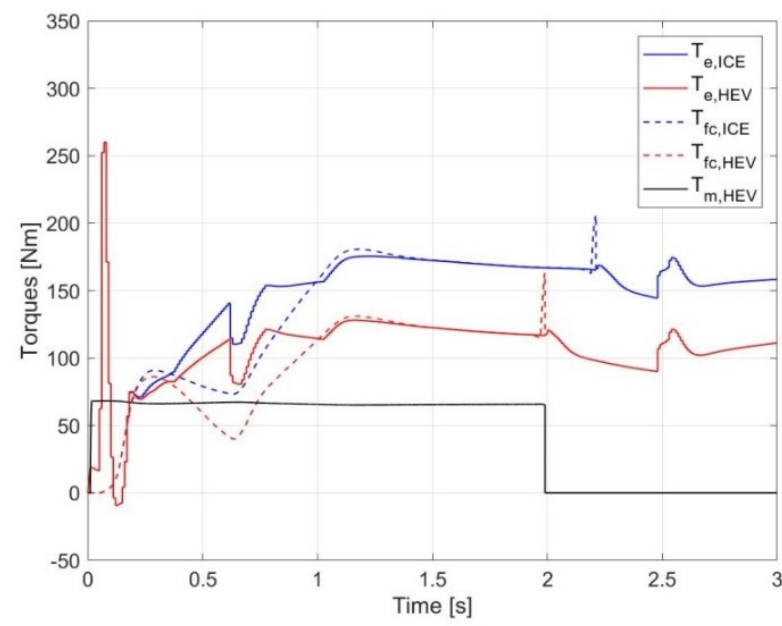

Figure 8 Second scenario: engine, clutch and electric motor torques reduced to the engine shaft, HEV mode (red lines), ICE mode (blue lines) and electric motor torque (black line).

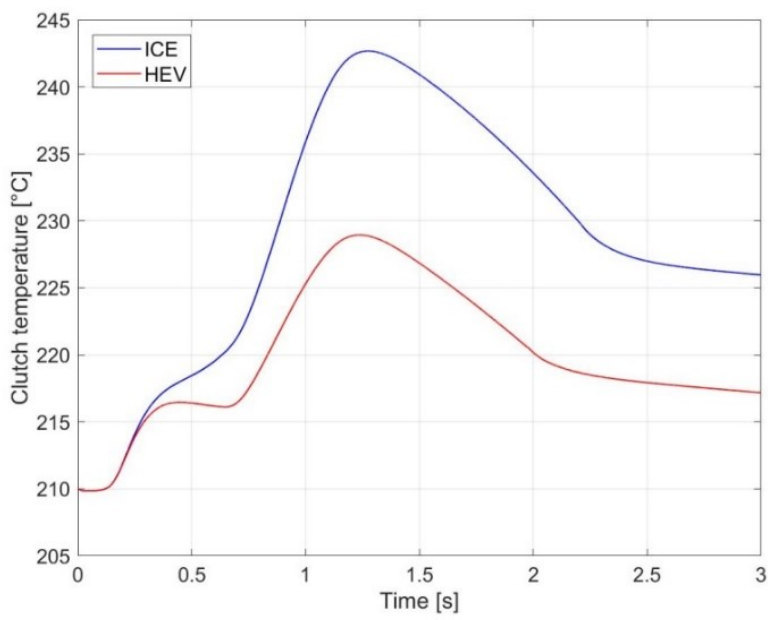

Figure 9 First scenario: clutch material temperature, HEV mode (red line), ICE mode (blue line).

underline lower temperature rise of clutch material in HEV mode: with the aid of EM, the reduction of clutch temperature attains $20 \%$ or even $45 \%$, according to the given scenarios. Under this light, the electric motor activation remarkably prevents clutch overheating after repeated engagements. Future analyses will embody more complex HEV model to include also the thermal evolution of the electric motor as well as the battery status to explore optimal trade-off between the temperatures of such components with the required thermal relief of dry clutch system. 


\section{Table of Figures}

Figure 1. Driveline model.

Figure 2. Estimated parameters: mean and standard deviation.

Figure 3. Control scheme.

Figure 4. First scenario: engine and clutch angular speeds, set point trajectories (black lines), HEV mode (red lines), ICE mode (blue lines).

Figure 5. First scenario: engine, clutch and electric motor torques reduced to the engine shaft, HEV mode (red lines), ICE mode (blue lines) and electric motor torque (black line).

Figure 6. First scenario: clutch material temperature, HEV mode (red line), ICE mode (blue line).

Figure 7. Second scenario: engine and clutch angular speeds, set point trajectories (black lines), HEV mode (red lines), ICE mode (blue lines).

Figure 8. Second scenario: engine, clutch and electric motor torques reduced to the engine shaft, HEV mode (red lines), ICE mode (blue lines) and electric motor torque (black line).

Figure 9. First scenario: clutch material temperature, HEV mode (red line), ICE mode (blue line). 
Mario Pisaturo received the M.Sc. and Ph.D. degrees in mechanical engineering from the University of Salerno, Italy, in 2010 and 2014, respectively. He is fellow of Department of Industrial Engineering, University of Salerno. The main research topic is: analysis theoretical/experimental of dry clutches for automotive with regard to automated manual transmissions and hybrid vehicles. He served as reviewer for several journals on the topics of Mechanics, Mechatronics, Tribology.

Adolfo Senatore received Ph.D. degree in Tribology from the University of Pisa, Italy, in 2002. He has been serving as Associate Professor at the Department of Industrial Engineering, University of Salerno, (2014current). Teacher of Mechatronics (2004-) and Mechanical Vibrations (2016-). His scientific work is documented by more than 190 scientific papers in the

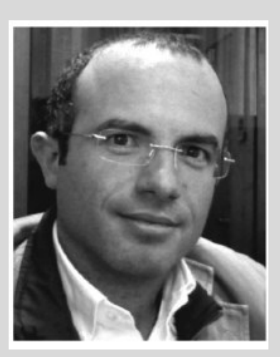
following areas: frictional modeling and model-based control of automotive transmissions, lubrication in internal combustion engines and journal bearings, innovative lubricants. He visited as Guest Professor the Department of Applied Mechanics of the Technische Universitat Berlin under DAAD program.

\section{References}

Chan, C. C. (2007) 'The State of the Art of Electric, Hybrid, and Fuel Cell Vehicles', Proceedings of the IEEE, 95(4), pp. 704-718. doi: 10.1109/JPROC.2007.892489.

D'Agostino, V., Senatore, A. and Pisaturo, M. (2013) 'Improving the engagement performance of automated dry clutch through the analysis of the influence of the main parameters on the frictional map', in 5th World Tribology Congress. Turin.

Feng, H., Yimin, M. and Juncheng, L. (2010) 'Study on heat fading of phenolic resin friction material for micro-automobile clutch', 2010 International Conference on Measuring Technology and Mechatronics Automation, ICMTMA 2010, 3, pp. 596-599. doi: 10.1109/ICMTMA.2010.386.

Glielmo, L. et al. (2006) 'Gearshift control for automated manual transmissions', IEEE/ASME Transactions on Mechatronics, 11(1), pp. 1726. doi: 10.1109/TMECH.2005.863369. 
Huang, Q., Song, J. and Li, L. (2011) 'Research on rapid testing platform for TCU of automated manual transmission', Proceedings - 3rd International Conference on Measuring Technology and Mechatronics Automation, ICMTMA 2011, 3, pp. 67-70. doi: 10.1109/ICMTMA.2011.588.

Oh, K. et al. (2007) 'Optimization of control strategy for a single-shaft parallel hybrid electric vehicle', Proceedings of the Institution of Mechanical Engineers, Part D: Journal of Automobile Engineering, 221(5), pp. 554-565. doi: 10.1243/09544070JAUTO93.

Pica, G. et al. (2016) 'Dry Dual Clutch Torque Model with Temperature and Slip Speed Effects', Intelligent Industrial Systems. Springer Singapore, 2(2), pp. 133-147. doi: 10.1007/s40903-016-0049-6.

Pisaturo, M., Cirrincione, M. and Senatore, A. (2015) 'Multiple constrained MPC design for automotive dry clutch engagement', IEEE/ASME Transactions on Mechatronics. doi: 10.1109/TMECH.2014.2335894.

Pisaturo, M. and Senatore, A. (2016) 'Simulation of engagement control in automotive dry-clutch and temperature field analysis through finite element model', Applied Thermal Engineering, 93, pp. 958-966. doi: 10.1016/j.applthermaleng.2015.10.068.

Pisaturo, M. and Senatore, A. (2017) 'Launch maneuver in Hybrid Vehicle: Electric Motor assistance to limit Temperature Rise in Automated Dry Clutch', in 21st International Conference on Mechatronics Technology. Ho Chi Minh City, Vietnam.

Pisaturo, M., Senatore, A. and D'Agostino, V. (2017) 'Could electric motor in HEV assist vehicle launch and relief dry clutch from thermal damage?', IEEE/ASME International Conference on Advanced Intelligent Mechatronics, AIM, pp. 327-333. doi: 10.1109/AIM.2017.8014038.

Pisu, P. and Rizzoni, G. (2007) 'A Comparative Study Of Supervisory Control Strategies for Hybrid Electric Vehicles', IEEE Transactions on Control Systems Technology, 15(3), pp. 506-518. doi: 10.1109/TCST.2007.894649.

Rakopoulos, C. D. and Giakoumis, E. G. (2009) Diesel Engine Transient Operation. Springer.

Sharifzadeh, M. et al. (2016) 'Vehicle tyre/road interaction modeling and identification of its parameters using real-time trust-region methods', IFAC-PapersOnLine. Elsevier B.V., 49(3), pp. 111-116. doi: 10.1016/j.ifacol.2016.07.019. 
Tribioli, L. et al. (2014) 'A real time energy management strategy for plugin hybrid electric vehicles based on optimal control theory', Energy Procedia. Elsevier B.V., 45, pp. 949-958. doi: 10.1016/j.egypro.2014.01.100.

Vagg, C. et al. (2016) 'Stochastic Dynamic Programming in the Real-World Control of Hybrid Electric Vehicles', IEEE Transactions on Control Systems Technology, 24(3), pp. 853-866. doi: 10.1109/TCST.2015.2498141.

Vasca, F. et al. (2011) 'Torque transmissibility assessment for automotive dry-clutch engagement', IEEE/ASME Transactions on Mechatronics, 16(3), pp. 564-573. doi: 10.1109/TMECH.2010.2047509.

Wang, J. et al. (2015) 'Hybrid electric vehicle modeling accuracy verification and global optimal control algorithm research', International Journal of Automotive Technology, 16(3), pp. 513-524. doi: 10.1007/s12239-015-0053-y.

Ye, X. et al. (2013) 'Modeling and control strategy development of a parallel hybrid electric bus', International Journal of Automotive Technology, 14(6), pp. 971-985. doi: 10.1007/s12239-013-0107-y.

\section{To cite this article:}

Pisaturo, M., \& Senatore, A., 2019. Electric motor and dry clutch control in launch manoeuvres of Mild-Hybrid vehicles based on AMT/DCT transmissions. Exchanges: The Interdisciplinary Research Journal, 7(1), 65-81. Retrieved from: http://doi.org/10.31273/eirj.v7i1.319. 